\title{
One Step Self-Calibration Procedure in Gamma-Ray Energy Measurements
}

\author{
Z. O. Guimarães-Filho and O. Helene \\ Instituto de Física, Universidade de São Paulo, \\ Caixa Postal 66318, CEP 05315-970, São Paulo, SP, Brazil
}

Received on 30 October, 2002

\begin{abstract}
This paper presents a one step self-calibration procedure, based on the least squares method, to be used in gamma-ray energy measurements. The obtained equation allows a qualitative interpretation of the information provided by an experiment.
\end{abstract}

\section{Introduction}

In the traditional calibration procedure used in gammaray energy measurements, the independent variables $x_{i}$, $i=1,2, \ldots, n$, corresponding to some well-known standard quantities (the dependent variables) $y_{i}$, are measured and a calibration function $y=f(x)$ is then fitted to the data.

The linear equation model of the fit is given by

$$
R=X_{C} \cdot A_{C}^{(0)}+\left(\varepsilon_{C}+\varepsilon_{R}\right)
$$

where $R$ is the column vector formed by the well known standard data, $X_{C}$ is the design matrix, and $\varepsilon_{C}$ and $\varepsilon_{R}$ are the unknown vector of errors due to the measurement and the standards, respectively. As is usual in experimental physics, we assume that

$$
\begin{aligned}
& \left\langle\varepsilon_{C}\right\rangle=0 \\
& \left\langle\varepsilon_{R}\right\rangle=0 .
\end{aligned}
$$

The covariance matrix is given by

$$
\left\langle\left(\varepsilon_{C}+\varepsilon_{R}\right)^{t}\left(\varepsilon_{C}+\varepsilon_{R}\right)\right\rangle=V_{C}+V_{R}
$$

where the errors of the standards and the measurements are supposed to be independent, $\left\langle\varepsilon_{C} \cdot \varepsilon_{R}\right\rangle=0$, and $V_{R}$ and $V_{C}$ are the covariance matrix of the standard and the measured data. In gamma-ray energy measurements, $V_{C}$ is given by

$$
\left(V_{C}\right)_{i i}=\left(\sigma_{i} . \tilde{b}\right)^{2}
$$

where $\tilde{b}$ is the estimate of the linear term of the energy calibration and $\sigma_{i}$ is the standard deviation of the peak position.

In a second step, some barely known gamma-ray energies, which were measured simultaneously with the standards, are obtained by interpolation using the fitted calibration function.

Usually the barely known quantities are not included in the calibration, since their statistical weights, proportional to $\left(\sigma_{j}\right)^{-2}$, are small if compared to the statistical weights of standard values. Also, the new interpolated values of the well-known quantities are usually not considered, perhaps because it is believed that their inclusion in the fit is difficult.

Ref. [1] discusses how to include both the standard data and the barely known data in the calibration and in the interpolation procedure. New and more precise values of physical quantities used as input data are determined using the information contained in the calibration curve. However, in Ref. [1], the procedure was developed in a two steps process and the difficulty with a singular covariance matrix was solved by using some special algorithms.

\section{One step self-calibration proce- dure}

In the self calibration procedure described here, the detector calibration and the energy interpolation is done in a single step. The least squares linear model equation is written as

$$
\left(\begin{array}{c}
0_{1} \\
R
\end{array}\right)=\left(\begin{array}{cc}
-I & X_{C} \\
I & 0_{2}
\end{array}\right)\left(\begin{array}{c}
A_{E}^{(0)} \\
A_{C}^{(0)}
\end{array}\right)+\left(\begin{array}{c}
\varepsilon_{C} \\
\varepsilon_{R}
\end{array}\right)
$$

where $A_{E}^{(0)}$ is the column vector formed by the true and unknown values of the energies, $I$ is the identity matrix, $0_{1}$ and $0_{2}$ are column and rectangular matrices with zeros, respectively. The covariance matrix corresponding to eq.(1) is

$$
V=\left(\begin{array}{cc}
V_{C} & 0_{2} \\
0_{2} & V_{R}
\end{array}\right)
$$

The self-calibration procedure is an application of the Least square method (LSM), without further assumptions. 
The results of the procedure therefore inherit all the properties of the LSM[2]: they are consistent, asymptotically unbiased, and are the minimum variance estimates.

\section{Discussion}

The improvement in input data precision by self-calibration deserves comment. It takes place because the apparatus gives some new information, determining some relations between input and the measured data. As a consequence, a measurement changes the data taken as standards and eq.(1) provides a new interpretation of the information given by an experiment.

Measurements of gamma-ray energies give some relations between the true (and unknown) values of the energies $\left(A_{E}^{(0)}\right)$ and the peak positions. These relations are given by

$$
0_{1}=-A_{E}^{(0)}+X_{C} \cdot A_{C}^{(0)}+\varepsilon_{C}
$$

and depend on the detector response function, included both in $X_{C}$ and $A_{C}^{(0)}$.

The previous knowledge $(R)$ of the gamma-ray energies is given by the equation

$$
R=A_{E}^{(0)}+\varepsilon_{R}
$$

Eq.(1) is just these last two equations written as a single one.

We must observe that the measured gamma-rays and the standards included in equations (2) and (3) can be different. In this case the proposed procedure allows the simultaneous update of the whole set of standards.

We would like to thank W. R. Carvalho Junior for a critical reading of the manuscript. This work was supported by FAPESP and CNPq.

\section{References}

[1] O. Helene, V. R. Vanin, and S. P. Tasi, Nucl. Instr. and Meth. A 433, 592 (1999).

[2] V. R. Vanin and P. Gouffon, "Tópicos Avançados em Tratamento Estatístico de Dados em Física Experimental”, LALIFUSP (1996). 